\title{
MedienPädagogik
}

Zeitschrift für Theorie und Praxis der Medienbildung

www.medienpaed.com

ISSN 1424-3636

Themenheft Nr. 37: Medienpädagogik als Schlüsseldisziplin in einer mediatisierten Welt. Perspektiven aus Theorie, Empirie und Praxis Herausgegeben von Henrike Friedrichs-Liesenkötter, Lara Gerhardts, Anna-Maria Kamin und Sonja Kröger

\section{Medienkritik und Medienpädagogik}

Horst Niesyto

\section{Zusammenfassung}

Medienkritik ist ein Grundbegriff der Medienpädagogik. Die Förderung von Medienkritik ist eine wesentliche Aufgabe der Medienpädagogik in allen pädagogischen Handlungsfeldern. Seit Bestehen der Medienpädagogik als wissenschaftliche Teildisziplin der Erziehungswissenschaft gibt es Definitionsangebote zum Begriff Medienkritik und Vorschläge zur Weiterentwicklung und Ausdifferenzierung des Begriffs. Im Unterschied hierzu entstanden nur relativ wenige empirische Studien, die die Untersuchung von Prozessen zur Förderung von Medienkritik in medienpädagogischen Handlungsfeldern zum Gegenstand hatten. Auch in der Theoriebildung gibt es Desiderata, insbesondere im Kontext der Digitalisierung und damit verbundenen Veränderungen in der gesellschaftlichen Kommunikation, der Sozialisation, der Bildung und Erziehung. Der folgende Beitrag bietet im ersten Teil einen Überblick zu bisherigen begrifflich-theoretischen Überlegungen, zu Praxisbeispielen und zu forschungsbezogenen Studien einer pädagogisch motivierten Medienkritik. Der zweite Teil greift ausgewählte Themen aus der aktuellen Fachdiskussion auf: die Notwendigkeit einer kritischen Medien- und Gesellschaftsanalyse, die Auseinandersetzung mit normativen Fragen und dem Subjektverständnis in der Medienpädagogik. Der Beitrag plädiert dafür, Medienkritik vor allem im Kontext einer politisch-kulturellen Medienbildung alltags- und praxisnah zu entwickeln. 


\title{
Media Criticism and Media Education
}

\begin{abstract}
Media criticism is a basic concept of media education. The promotion of media criticism is an essential task of media education in all pedagogical fields of practice. Since media education was established as a scientific sub-discipline of educational science, there have been offers for definitions of the term media criticism and suggestions for the further development and differentiation of the term. In contrast, relatively few research studies investigated processes for the promotion of media criticism in media educational fields of practice. Nevertheless, there are also desiderata in the field of theory, especially in the context of digitalization and associated changes in the areas of social communication, socialization, and education. In the first part, the following article provides an overview of previous conceptual-theoretical considerations, practical examples and research-related studies of a pedagogically motivated media criticism. The second part takes up selected topics from the current expert discussion: the necessity of a critical media and social analysis, the reflection of normative questions and the understanding of the subject in media education. The article argues in favour of developing media criticism in a practical way and close to everyday life, especially in the context of political-cultural media education.
\end{abstract}

\section{Medienkritik in pädagogischen Kontexten - bisherige Konzepte, Praxis und Forschung ${ }^{1}$}

Der erste Teil dieses Beitrags konturiert den Gegenstand von Medienkritik. Deutlich wird, dass sich Medienkritik auf ein breites Spektrum von Themen und Disziplinen bezieht. Im Hinblick auf eine pädagogische Medienkritik werden theoretische Konzepte, Praxisbeispiele und Forschungsbeiträge im Überblick dargestellt.

1 Der Beitrag knüpft an den Sammelband Medienkritik im digitalen Zeitalter (Niesyto und Moser 2018) sowie an Diskussionen auf einem Austauschtreffen an, zu dem die Herausgeber des Sammelbands im März 2019 eingeladen hatten. Das Austauschtreffen fand im Anschluss an die Frühjahrstagung der Sektion Medienpädagogik in der Deutschen Gesellschaft für Erziehungswissenschaft (DGfE) an der Universität Paderborn statt (siehe: https://kw.uni-paderborn.de/mp2019/ sowie http://medienkritik-lb.de/austauschtreffen/). 


\section{Zum Gegenstand von Medienkritik}

Medienkritik ist im wissenschaftlichen Kontext ein Begriff, der in verschiedenen Disziplinen verwendet wird, insbesondere in der Medien- und Kommunikationswissenschaft, der Publizistikwissenschaft und der Medienpädagogik. Er bezieht sich vom Gegenstand her vor allem auf die Auseinandersetzung mit produkt- bzw. werkbezogenen Medienangeboten (z.B. Literaturkritiken, Fernsehkritiken, Filmkritiken, Netzkritiken), auf professionelle Qualitäts- und Medienstrukturfragen (z.B. Unterscheidung zwischen Information und Meinung, Pluralismusgebot, medienethische Grundsätze), auf gesellschafts- und systemkritische Dimensionen (z.B. ökonomische Abhängigkeiten und Konzentrationsprozesse, Datenkapitalismus, Technologiekritik, Datenkontrolle und politische Machtstrukturen) sowie die (Selbst-)Reflexion der Mediennutzung in unterschiedlichen lebensweltlichen und soziokulturellen Kontexten (z.B. vorhandene Fähigkeiten zu einer kritisch-reflexiven Mediennutzung in unterschiedlichen Altersgruppen und Milieus, Formen exzessiver Mediennutzung, handlungsorientierte Medienkritik in Medien- und MakerSpace-Projekten).

Hans-Dieter Kübler weist in einem Überblicksbeitrag zu Prämissen und Paradigmen von Medienkritik (2018) darauf hin, dass sich in der Mediengeschichte

«unzählige Beispiele, Kategorien und Schwerpunkte von Medienkritik [finden], die sich zunächst als recht umfängliche, sodann aber auch als fundamentale Zugangsweise zu stets sich verändernden Medienwelten ausweist. Demnach vervielfältigt sich Medienkritik jeweils in den gegebenen Settings, definiert neue Ziele und Herausforderungen und konturiert entsprechend ihre Aufgaben.» (Kübler $2018,18)$

Kübler unterstreicht die Relevanz normativer Dimensionen jeglicher Formen von Medienkritik, die je nach medialem Gegenstandsbereich, historisch-gesellschaftlichen Kontexten und Urheber sehr heterogen oder auch widersprüchlich ausfallen (ebd., 18). Medienkritik ist «eine zwar transparente, aber eher normativ ausgerichtete Beschäftigung und Beurteilung von Medien, die auf wertbegründete Prinzipien und/oder Ziele rekurriert» (ebd., 15). Diese Feststellung ist 
«insbesondere für eine pädagogisch motivierte Medienkritik (Kübler 2006) relevant, da sie nicht nur die Medienwelt beurteilen und bewerten, sondern auch ihre Adressaten befähigen will, dies ebenfalls tun zu können. Ziele und Methoden stehen daher unter besonderer Prüfung.» (Kübler 2018, 15)

Der Autor äussert sich zur Erreichung der Ziele einer pädagogisch motivierten Medienkritik in seinem Beitrag aus dem Jahre 2006 eher zurückhaltend (siehe der nächste Teil) und betont in einem aktuellen Beitrag aus einer primär medienwissenschaftlichen Perspektive vor allem die theoretischen Desiderata einer zeitgemässen Medienkritik unter den Bedingungen der Digitalisierung und global agierender Netzwerk-Konzerne (Kübler 2018).

\section{Theoretische Konzepte einer pädagogisch motivierten Medienkritik}

Im Kontext von Film- und Medienerziehung haben sich viele Pädagoginnen und Pädagogen jahrzehntelang den jeweils neuen Medien mit jugendschützenden Vorbehalten im Sinne einer bewahrpädagogischen Kultur- und Medienkritik genähert. Die Intention war es, Kinder und Jugendliche vom 〈passiven Medienkonsum〉 abzubringen und sie vor 〈verderblichen〉 Medienwirkungen zu bewahren. Dieter Baacke resümierte hierzu: «Die Nähe des pädagogischen Räsonnements zu einer eher medienabwehrenden Kulturkritik ist daher Tradition und kennzeichnet ein immer wieder gestörtes Verhältnis» (Baacke 1997, 29). Baacke betonte vor allem die mit dieser Form der Kulturkritik verknüpfte normative Kontrollorientierung, die Kinder und Jugendliche letztlich als unmündige Rezipienten behandelt (ebd.).

Die wissenschaftlich fundierte Medienpädagogik hat sich stets differenziert und kritisch mit medienbezogenen Problemlagen und Aufgaben der Förderung von Medienkompetenz auseinandergesetzt (Aufenanger 2018). ${ }^{2}$ So entwickelte Baacke Mitte der 1990er Jahre im Rahmen des handlungsorientierten Ansatzes ein Medienkompetenz-Modell, welches einen

2 Siehe hierzu Aufenanger 2018 sowie Niesyto 2018 (Niesyto 2018, 59-61) zu den historischen Entwicklungslinien der Medienpädagogik bezüglich Medienkritik. 
nachhaltigen Einfluss auf die Theorie und Praxis der Medienpädagogik hat. Nach diesem Modell ist Medienkritik neben Medienkunde, Mediennutzung und Mediengestaltung einer der vier grundlegenden Medienkompetenzbereiche. Baacke differenzierte Medienkritik in drei Dimensionen:

- die analytische Dimension umfasst die Fähigkeit, problematische gesellschaftlich-mediale Prozesse angemessen zu erfassen;

- die reflexive Dimension bezeichnet die Fähigkeit, dass Menschen das analytische Wissen auf sich selbst und ihr Medienhandeln anwenden können;

- die ethische Dimension verknüpft das analytische Denken und den reflexiven Rückbezug im Hinblick auf ein gesellschaftlich und sozial verantwortliches Handeln (Baacke 1997, 98).

Baacke betrachtete Medienkritik als eine zu Medienkunde, Mediennutzung und Mediengestaltung quer liegende Kompetenz und merkte an, dass ein Mangel des Begriffs Medienkompetenz «seine pädagogische Unspezifität» sei (ebd., 99). Baackes Schlussfolgerung ist, auch in Zusammenhang mit Überlegungen zu den Begriffen Erziehung und Bildung:

«Wer von Medienkompetenz redet, muß also gleichzeitig davon reden, wie diese zu vermitteln sei und wo das Subjekt in seiner sich ausbildenden oder sich ausgebildet habenden Selbstverantwortlichkeit seinen kommunikativen Status bestimmt.» (ebd., 100)

Damit hat Baacke ein Desiderat benannt, das in der Folgezeit u.a. zur Unterscheidung zwischen vorhandenen und wünschenswerten Medienkompetenzen und zu verschiedenen theoretisch-konzeptionellen Überlegungen zur Förderung einer pädagogisch motivierten Medienkritik im Kontext einer handlungsorientierten Medienpädagogik führte.

So verwendete Hans-Dieter Kübler den Begriff «pädagogische Medienkritik> mit den Dimensionen Medienwissen, Medienanalyse (Methoden), Medienrezeption/Reflexivität, Medienbewertung - jeweils mit weiteren Subkategorien (Kübler 2006, 44-46). Sonja Ganguin untersuchte in einer empirischen Analyse zur Begriffsbestimmung und Evaluation von Medienkritik die Definitionsangebote zu Medienkompetenz von Stefan Aufenanger, Dieter Baacke, Heinz Moser und Gerhard Tulodziecki. Sie 
entwickelte eine Neukonzeptualisierung von Medienkritik in medienpädagogischen Kontexten (Ganguin 2006, 71-74):

- Wahrnehmungsfähigkeit (Raum-, Zeit- und Sinneswahrnehmung) als Voraussetzung, um Medien und ihre Strukturen, Inhalte, Gestaltungsformen, Wirkungsmöglichkeiten und Entwicklungen zu erkennen und zu durchschauen;

- Decodierungsfähigkeit hinsichtlich der verschiedenen «Mediensprachen〉 und den damit verbundenen Codes, Symbolen, Mustern und Gedächtnisleistungen;

- Analysefähigkeit als Unterscheidungs- und Klassifikationsfähigkeit bezüglich unterschiedlicher Medieninhalte, Medienformate und Genres;

- Reflexionsfähigkeit im Sinne von Distanzierungsfähigkeit und Wechsel der Perspektive;

- Urteilsfähigkeit bezüglich einzelner Medienangebote und der Medienentwicklung auf der Grundlage bestimmender und reflektierender Urteilskraft sowie objektiv-medienbezogener (z.B. Stichhaltigkeit, Ausführlichkeit) und subjektiver Kriterien (z.B. Geschmackspräferenzen).

Die Stärke des Konzepts von Ganguin liegt in einer kategorialen, kriteriengeleiteten Differenzierung von Teildimensionen. Aspekte des historisch-gesellschaftlichen Wandels von Medienentwicklungen und medienkulturellen Praktiken und deren Bedeutung für Medienkritik wurden bislang eher am Rande thematisiert. ${ }^{3}$ In späteren Veröffentlichungen legten Ganguin und Sander einen besonderen Wert auf alters-/entwicklungsbezogene Phasen als Hintergrund für medienkritisches Handeln in Anlehnung an entwicklungspsychologische Studien von Kohlberg und Piaget: die Aneignungsphase, die Kritische Phase und die Reifungsphase (Ganguin und Sander 2015, 237-44). Auch Gerhard Tulodziecki und Silke Grafe rekurrieren in ihrem handlungs- und entwicklungsorientierten Konzept von Medienkritik auf die Modelle von Kohlberg und Piaget (Tulodziecki und Grafe 2018). Dorothee Meister und Uwe Sander formulieren vor dem Hintergrund einer empirischen Studie zur Werbekompetenz von Kindern

3 Der aktuelle Beitrag von Ganguin/Gemkow/Haubold im vorliegenden Band, der sich vor allem mit medienkritischer Decodierungsfähigkeit auseinandersetzt, erweitert die bisherige Perspektive und bezieht sich explizit auf Decodierungsfähigkeit in digitalen Kontexten. 
u.a. die These, dass sich Werbekompetenz bei Kindern zunächst in einer «vorkritischen» Form entwickelt und dann mit zunehmenden Alter und Bildungsgrad zunimmt (Meister und Sander 2017, 191).

Alle Konzepte betonen kognitiv-analytische Prozesse und Fähigkeiten. Kübler unterstreicht, dass kognitive Fähigkeiten «erwünscht bzw. anzustreben» sind im Sinne analytisch-methodischer Fähigkeiten zur Medienkritik. Er weist aber zugleich darauf hin, dass es «didaktisch-pädagogisch ein recht weiter Weg»sei, um «gewisse Niveaus der Distanzierung und Abstraktion» zu erreichen, insbesondere hin zu einem «Urteil, das objektive, genrespezifische Bezüge und differenzierte Qualitätsaspekte umfasst» (Kübler 2006, 43).

\section{Medienkritik in pädagogischen Handlungsfeldern}

In der medienpädagogischen Praxis standen stets handlungsbezogene Ansätze der Medienpädagogik im Vordergrund, die sich mit unterschiedlichen theoretisch-konzeptionellen Akzentsetzungen und praxisfeldbezogenen Schwerpunkten ausdifferenzierten. Sich mit Medien kreativ und kritisch zu artikulieren, wurde zum Leitmotiv zahlreicher medienpädagogischer Aktivitäten. Im Unterschied zu einer Medienschelte, die als Alternative zu problematischen Mediennutzungsformen «medienfreie) Aktivitäten empfiehlt, fördert die Medienpädagogik den aktiv-produktiven, kritischen und sozial verantwortlichen Umgang mit Medien (Niesyto, Rath, und Sowa 2006). Hierzu gehören nicht nur Aktivitäten im präventiven Kinder- und Jugendmedienschutz, sondern ein breites Spektrum medienkritischer Analysen und Aktivitäten. Sie reichen von der Auseinandersetzung mit stereotypen Rollenbildern in Medien über das Entdecken medialer Ton- und Bildmanipulationsmöglichkeiten (z.B. Holzwarth 2012) bis hin zu reflexiven Phasen in Projekten aktiver Medienarbeit und der kritischen Prüfung und Bewertung verschiedener Medienprodukte durch Kinder und Jugendliche im Rahmen von Filmjurys (z.B. spinxx.de) und der Entwicklung von Spielkritiken bei der gemeinsamen Erprobung von Computerspielen (z.B. spielbar.de; spielratgeber-nrw.de). Im Kontext handlungsorientierter Ansätze ist es dabei wichtig, von den jeweils subjektiven Medienerfahrungen auszugehen und als Pädagogin und Pädagoge durch gute Fragen und 
Aufgabenstellungen Reflexionsprozesse zu fördern - gerade im Hinblick auf die Auseinandersetzung mit (medien-)ethischen und (sozial-)moralischen Orientierungen, Ambivalenzen und Dilemma- bzw. Konfliktsituationen (vgl. hierzu Tulodziecki und Grafe 2018, 133-35).

In den vergangenen 15 Jahren stand die Auseinandersetzung mit digitalen Medien, Internetkommunikation und mobilen Medien im Vordergrund. Mit Blick auf die kritisch-reflexiven Potentiale werden u.a. die Kritik-, Ergänzungs- und Kommentierungspraktiken (z.B. bei Weblogs), die Notwendigkeit von Quellenkritik und Kontextualisierung von Informationen (z.B. bei Wikipedia), ein selbstkontrollierter Umgang mit eigenen Daten (z.B. handysektor.de, klicksafe.de, webhelm.de, webdaysmooc) und die kreativen Möglichkeiten digitaler Medienproduktion, z.B. für das Erzählen von Geschichten (Digital Storytelling), oder kritische und subversive mediale Inszenierungen von Medienaktivisten, Hacker- und Gaming-Kulturen betont (u.a. Hug 2011; Missomelius 2018). ${ }^{4}$

Die Aneignung von kritischem Strukturwissen zu algorithmischen Prozessen wird aktuell zu einer zentralen Aufgabe pädagogisch motivierter Medienkritik (Knaus 2018; Maurer und Ingold 2018). Thomas Knaus bezeichnet die gesellschaftliche Entmystifizierung von Technik und informatischen Prozessen als ein wichtiges Desiderat im Sinne der Entwicklung einer kritischen Haltung gegenüber Algorithmen, Codes und den von ihnen erzeugten Daten: «Ein Grundverständnis für Technik bzw. technische und informatische Prozesse wird zur essentiellen Voraussetzung dieser erweiterten Medienkritik» (Knaus 2018, 99-100). Damit ist nicht gemeint, «dass jeder Mensch 〈coden〉 oder 〈programmieren〉 können sollte [...], gemeint ist die Fähigkeit zur kritischen Analyse jeglicher medialer und technischer Artefakte sowie die Kompetenz, deren Provenienz und Anspruchsgruppen zu hinterfragen und durchschauen zu können - idealerweise anhand des selbstverantwortlichen technischen und medialen Handelns.» (Knaus 2018, 100)

4 Siehe auch eine aktuelle Übersicht zu ausgewählten Projekten und Praxisbeispielen zum Thema Medienkritik in Niesyto 2019a (spezielle PDF zum Beitrag). 
Die pädagogisch spannende Frage ist, wie dieses Strukturwissen entstehen kann. Hier ist die These, dass ein solches Strukturwissen nicht allein kognitiv angeeignet werden kann, sondern dass es eigener medienund sozialästhetischer Erfahrungsprozesse bedarf (siehe in diesem Beitrag den Teil zum Themenaspekt (Medienkritik und pädagogisches Handeln〉).

\section{Medienkritik als Thema medienpädagogischer Forschung}

Es gibt bis dato keine wissenschaftliche Studie, die schwerpunktmässig Fragen der Medienkritik im Kontext handlungsbezogener medienpädagogischer Praxisaktivitäten breiter und systematisch untersucht. Allenfalls liegen zu einzelnen Aspekten Befunde vor, die sich z.B. auf die Frage beziehen, inwieweit im Rahmen der Förderung von Eigenproduktionen mit Medien auch Reflexionen stattfinden. Hier stellt z.B. die Studie Medienpädagogik und soziokulturelle Unterschiede (Niesyto 2004) fest, dass die Reflexion der Praxis beim Erstellen der Eigenproduktionen oft zu kurz kommt. Lediglich ein Drittel der in Experteninterviews befragten 67 Mitarbeiterinnen und Mitarbeiter (in Baden-Württemberg und Rheinland-Pfalz) gab an, dass regelmässig Auswertungen und Reflexionen in Projekten aktiver Medienarbeit stattfinden (ebd., 104). Nur zwei Personen sagten, dass sie regelmässig Reflexionen zur Projektpraxis im Kreis der Mitarbeiterinnen und Mitarbeiter der jeweiligen Einrichtung vornehmen (ebd., 105).

Mit Blick auf die alltägliche Mediennutzung Jugendlicher untersuchte eine Bielefelder Studie - in Anlehnung an das Medienkompetenzmodell von Baacke - das Medienhandeln Jugendlicher und machte auch die Fähigkeit zur Medienkritik und Medienreflexion Jugendlicher zum Gegenstand (Treumann u.a. 2007). ${ }^{5}$ Die Studie schlussfolgert aus den Befunden u.a., dass das Medienhandeln der befragten Jugendlichen in ihre konkreten Weltdeutungen eingebettet ist. Als pädagogische Empfehlung wird u.a. formuliert, dass Medienkritik nicht per se als erwartbare Festlegung an Jugendliche heranzutragen ist, sondern vor dem Hintergrund

5 In der Panoramastudie wurden Interessen, Neigungen und Medienkompetenzen bei 12- bis 20-Jährigen erhoben. Methodisch basierte die Studie auf einer Fragebogenerhebung (über 3000 Heranwachsende), die mit qualitativen Leitfadeninterviews und Gruppendiskussionen verknüpft wurde (prototypische Auswahl von Jugendlichen). 
der «spezifisch generierten kollektiven Orientierungs- und Deutungsrahmen Jugendlicher» hergeleitet werden kann (ebd., 669). Dieser Befund unterstreicht die Notwendigkeit, Medienkompetenz nicht einseitig aus Expertensicht zu definieren, sondern stets auch im Blick zu haben, welche Medienerfahrungen und Medienkompetenzen Kinder, Jugendliche und Erwachsene mitbringen und wie in diesem Rahmen Impulse zur reflexiven Auseinandersetzung mit vorhandenen Orientierungs- und Deutungsmustern gegeben werden können. ${ }^{6}$

Bezüglich der Operationalisierbarkeit der Kategorie 〈Medienkritik〉 hielten die Autorinnen und Autoren fest:

«Was die Kompetenz-Dimension der Medienkritik angeht, so scheint sie mit quantitativen Methoden nicht befriedigend rekonstruierbar zu sein. Um sie angemessen erfassen zu können, empfehlen wir angesichts der außerordentlichen Komplexität dieser Dimension die Anwendung nichtstandardisierter Methoden.» (Treumann u.a. 2007, 191)

Im Laufe der Fachdiskussion zur weiteren Professionalisierung der medienpädagogischen Ausbildung an Hochschulen entstanden einzelne Evaluationsstudien, die auch Medienkritik als Teilaspekte umfassen. So stellt z.B. eine Studie, die an der Pädagogischen Hochschule Zürich entstand, u.a. fest, dass «an der Spitze der für die Studierenden wichtigsten Inhalte Überlegungen zu den Chancen und Risiken der Mediennutzung (Rang 1) und zu deren Einfluss auf Kinder und Jugendliche (Rang 2)» stehen (Scheuble, Signer, und Moser 2014, 120). Die Autorinnen und Autoren folgern aus der Untersuchung u.a.: «Gerade die Reflexion auf Medien, die mit den Schülerinnen und Schülern zusammen erreicht werden soll, setzt voraus, in solchen Fragen über einen fundierten Kenntnisstand zu verfügen» (ebd., 122) und in diesem Kontext eine medienpädagogische Grundbildung im Sinne von Basiskompetenzen als Mindeststandards für die Studierenden zu fördern.

In der Evaluation des Entwicklungsprojekts Digitales Lernen Grundschule - Stuttgart/Ludwigsburg, in welchem 215 Studierende der Pädagogischen Hochschule Ludwigsburg über 50 Unterrichtserprobungen an

6 Siehe in diesem Kontext auch die Aussagen zur Bedeutung der Subjektspezifik für die Medienkritik im Beitrag von Ganguin/Gemkow/Haubold im vorliegenden Band. 
einer Grundschule in Stuttgart durchführten, wurde bezüglich kritischreflexiver Impulse im Kontext von Medienproduktionen u.a. festgestellt, dass Schülerinnen und Schüler von Studierenden zu wenig Impulse erhielten, um insbesondere aus dem Modus der Produktion heraus über die selbst erstellten Medienprodukte nachzudenken und sich mit der Qualität der eigenen Materialien auseinanderzusetzen. Die Beobachtungen und Befunde verdeutlichen, dass medienästhetische und -kritische Kompetenzen wichtig sind, damit Studierende bei digitalen Medienproduktionen, die Schülerinnen und Schüler erstellen, situationsbezogen Fragen, Hinweise und Reflexionen integrieren können. Digitale Technik und digitale Ästhetik verändern zwar bisherige analoge Zeichen- und Symbolsysteme, sie ersetzen diese aber nicht. Gerade im Hinblick auf Medienkritikfähigkeit sind nach wie vor grundlegende Kenntnisse zu auditiven, visuellen und audiovisuellen Ausdrucksformen notwendig (Niesyto 2019).

In einer Dissertationsschrift über Medienaneignung und ästhetische Werturteile setzt sich Niels Brüggen mit den Fragen auseinander, welche Bedeutung ästhetische Urteile im Prozess der Medienaneignung haben und inwiefern das Gefallen oder Nichtgefallen intersubjektiv begründet werden kann (Brüggen 2017). Damit werden Fragen behandelt, die auch für eine pädagogisch motivierte Medienkritik relevant sind. In Erweiterung des medienpädagogischen Konzepts der Medienaneignung (Schorb und Theunert 2000) entwickelte Brüggen grundlagentheoretische Überlegungen zu ästhetischen Werturteilen. Mit Blick auf die medienpädagogische Praxis und weitere Forschungsarbeiten weist er in Zusammenhang mit Erfahrungen aus Web 2.0-Werkstätten auf Forschungs-Desiderata hin, z.B. zur Frage, «inwiefern Partizipationshürden auch durch Werteigentümlichkeiten in den Angeboten entstehen können» (gemeint sind Foto- und Video-Plattformangebote, Brüggen 2017, 258).

Christian Seyferth-Zapf et al. arbeiten an einer empirischen Studie zum Themenaspekt 〈mediale Propaganda〉. Untersucht wird auf der methodologischen Grundlage einer gestaltungsorientierten Bildungsforschung (Tulodziecki, Grafe, und Herzig 2013) ein «medienpädagogisches Konzept zur Förderung von Medienkompetenz unter besonderer Berücksichtigung der Diskussion internationaler Propaganda im Rahmen eines deutsch-amerikanischen Dialogs» (Seyferth-Zapf, Seyferth-Zapf, und Grafe 
2019). Hintergrund ist die Problemlage, dass in den letzten Jahren durch Social Bots, Fake News etc. neue Propagandaformate und -mechanismen entstanden sind, die auch eine Herausforderung für die pädagogische Medienkritik darstellen. Im Rahmen der explorativen Fallstudie konnte z.B. bezüglich des Inhaltsbereichs «Medieneinflüsse auf Individuum und Gesellschaft〉 festgestellt werden, «dass je nach persönlichem soziokulturellen Kontext Propaganda interindividuell ganz unterschiedliche Gefühle und Assoziationen auslösen kann» (ebd.). Die Autorinnen- und Autorengruppe betont u.a., dass künftige Forschungen zu dem Themenaspekt nicht nur rezeptive und interaktive Nutzungsformen, sondern auch die Eigenproduktion medialer Angebote umfassen sollten (ebd.).

In Zusammenhang mit aktuellen Bestrebungen, Medienbildung und Elemente informatischer Bildung an Grundschulen stärker zu verankern, entstand an einer Schweizer Primarschule ein MakerSpace-Projekt. In dem Projekt werden analoge und digitale Werkstoffe in handlungsorientierter Perspektive zum Gegenstand des obligatorischen schulischen Unterrichts. Im Rahmen einer Evaluationsstudie, die sich an dem Forschungsansatz 〈Design-Based-Research〉 orientiert, untersuchen Selina Ingold und Björn Maurer u.a. die Möglichkeiten, eine produkt- und wertebezogene Kritikund Reflexionsfähigkeit bei den Schülerinnen und Schülern zu fördern. Ein Zwischenbericht bilanziert Chancen, aber auch Grenzen einer wertebezogenen Reflexionskompetenz:

«Die Reflexionen gingen insgesamt jedoch nicht über das konkrete Produkt und die damit verbundenen technischen und ästhetischen Fragestellungen hinaus. Haltbarkeit oder ökologische Nachhaltigkeit waren hier kaum ein Thema. Fragen zur gesellschaftlichen Wirkung im Sinne einer weiterreichenden Technikfolgenabschätzung ergaben sich nicht.» (Maurer und Ingold 2019, 51)

Die Arbeitsgruppe des Projekts zog vor dem Hintergrund der ersten Erfahrungen Konsequenzen für das anstehende Re-Design des Projekts, das prozessbegleitend vorgenommen wird (ebd., 52-53). So soll künftig verstärkt an Themen der Bildung für nachhaltige Entwicklung (UN-Ziele) angeknüpft und zusammen mit den Schülerinnen und Schülern Produktbeurteilungskriterien für die Reflexionsrunden erarbeitet werden. 
Es ist zu hoffen, dass den skizzierten Beispielen weitere Studien und Forschungen zu Fragestellungen einer pädagogisch motivierten Medienkritik folgen. Wünschenswert sind vor allem Studien in pädagogischen Handlungsfeldern, die Entwicklungsprozesse auf der Basis qualitativer Methoden untersuchen. Wie Treumann et al. in Zusammenhang mit ihrer breit angelegten empirischen Studie festhalten, lässt sich die KompetenzDimension der Medienkritik mit quantitativen Methoden nicht befriedigend fassen (Treumann u.a. 2007, 191).

\section{Medienkritik: Ausgewählte Themen aus der aktuellen Fachdiskussion}

Wie bereits in früheren Beiträgen ausgeführt (u.a. Niesyto 2017), trübte sich in den 1990-er Jahren und in der ersten Dekade der 2000-er Jahre in Zusammenhang mit einer Präferenz für subjekt- und kulturtheoretisch motivierte Medienaneignungsstudien der Blick für kritische Analysen zu gesellschaftlich-medialen Transformations- und Formatierungsprozessen und ihren Einfluss auf Mediensozialisation und Medienhandeln. Die Förderung von Medienkritik blieb zwar in den diversen Medienkompetenzmodellen weiterhin eine Zielgrösse und es gibt diverse Praxisprojekte, in denen die Förderung von Medienkritik eine wichtige Rolle spielt. Im gesamten medienpädagogischen Theoriediskurs fristete das Thema «Medienkritik〉 jedoch lange Zeit eher ein Schattendasein. Die Verschärfung medialer, sozialer und bildungsbezogener Problemlagen in der Gesellschaft führte in den letzten Jahren wieder zu einer stärkeren Berücksichtigung medienkritischer Fragestellungen, insbesondere was eine kritische Medien- und Gesellschaftsanalyse und damit verknüpfte Aufgaben für medienpädagogisches Handeln betrifft.

Der folgende Teil fokussiert auf Fragen einer kritischen Medien- und Gesellschaftsanalyse, auf das Subjektverständnis und auf normative Fragen bezüglich Medienkritik. Mit Blick auf praktisches medienpädagogisches Handeln plädiert der Beitrag im Schlussteil für die Stärkung von Ansätzen einer politisch-kulturellen Medienbildung, die die Förderung von Medienkritikfähigkeit in einem engen Wechselverhältnis von Persönlichkeitsbildung und gesellschaftlicher Teilhabe verortet. 


\section{Notwendigkeit einer kritischen Medien- und Gesellschaftsanalyse}

Aktuelle medienpädagogische Diskurse und Publikationen befassen sich verstärkt mit Fragen einer kritischen Medien- und Gesellschaftsanalyse. Zu nennen sind vor allem Analysen zur Exklusion des Humanen im Digitalzeitalter (u.a. Damberger 2019), zum digitalen Kapitalismus (u.a. Niesyto 2017), zur Datenkritik (u.a. Dander 2017; Aßmann u.a. 2016) und zur Technikkritik (u.a. Knaus 2017), zur digitalen Ungleichheit (u.a. Verständig, Klein, und Iske 2016) und zum digitalen Strukturwandel von Öffentlichkeit (u.a. Barberi und Swertz 2017; Röll 2018; Verständig 2017). Ein wichtiges Thema ist auch die Auseinandersetzung mit digitaler Ethik, den normativen und wertbezogenen Grundlagen und Kriterien für die Reflexion und Bewertung von Prozessen des digitalen Wandels (u.a. Grimm und Zöllner 2018; Rath 2017). Die Analysen verdeutlichen zum einen die Komplexität und die Vielzahl von Fragestellungen, Phänomenbereichen und Strukturen des digitalen Wandels. So ist es z.B. angesichts komplexer Plattformen bei Hybridmedien ein Problem, isolierte Analysen zu Einzelmedien und zu entsprechenden Medienkritiken zu machen.

Es geht dabei nicht nur um die technologische Ebene, sondern auch um Veränderungsprozesse bezüglich gesellschaftlicher Kommunikation, Sozialisation, Bildung und Kultur. Unstrittig ist, dass das exponentielle Wachstum von digitalen Speicherkapazitäten, die Miniaturisierung von Einzelbausteinen, die mobile Einsetzbarkeit und die interaktiven Möglichkeiten in Echtzeitkommunikation strukturelle Eigenschaften digitaler Medien sind, welche einen enormen Schub in der Kommunikation ermöglicht haben. Gleichwohl ist die Digitalisierung in nahezu allen Bereichen des gesellschaftlichen Lebens nicht allein technologieinduziert, sondern wird von ökonomischen und politischen Interessen massiv forciert. Es mangelt derzeit an einer zeitgemässen Reformulierung einer politökonomischen Medien- und Gesellschaftsanalyse, die medial-gesellschaftliche Strukturmuster und Interessenkonstellationen herausarbeitet und diese mit aneignungstheoretischen Ansätzen des subjektiven Medienhandels verbindet (Vermittlung von Mikro- und Makro-Ebene).

Der Analyseansatz einer «tiefgreifenden Mediatisierung» im Kontext eines «kommunikativen Konstruktivismus» und «kommunikativer Figurationen» (Hepp 2018) weist zwar auf wichtige medienbezogene 
Entwicklungstrends (Ausdifferenzierung, Konnektivität, Omnipräsenz, Innovationsdichte, Datafizierung), den Stellenwert digitaler Materialität und auf die Überwindung der Einzelmedienperspektive hin und betont, «dass die grundlegenden Elemente der Konstruktion der sozialen Wirklichkeit selbst medial vermittelt sind» (Hepp 2018, 7). Der Ansatz erwähnt aber nur am Rande die Notwendigkeit, eine «politische Ökonomie von Infrastruktur» zu untersuchen (unter Hinweis auf eine «umfassende Privatisierung» von «Tech-Unternehmen») (ebd., 9). Die von Hepp ins Zentrum weiterer Theorie- und Forschungsarbeit gerückte «materialistische Phänomenologie» betont sehr stark die Veränderung gesellschaftlicher und kommunikativer Beziehungen durch technische Netzwerke und Algorithmen, thematisiert jedoch weniger Macht- und Herrschaftsfragen, die mit der Nutzung dieser Technologie verbunden sind.

Der Ansatz der strukturalen Medienbildung von Benjamin Jörissen und Winfried Marotzki (2009) bezieht Strukturfragen im Wesentlichen auf die immanente Strukturiertheit der Medien. Es geht um die konstitutiven Aspekte von Medien («Medialität») und um «transgressive Artikulationen im Spannungsfeld von Ästhetik und Medialität» (Jörissen 2015, 55-62), die ästhetische Differenzerfahrungen und ästhetische Selbstbestimmung intendieren. Analytische Bezüge zu gesellschaftlichen und ökonomischen Strukturfragen, insbesondere im Hinblick auf Macht- und Herrschaftsinteressen, sind bislang kein konstitutiver Bestandteil des Ansatzes der strukturalen Medienbildung. Im Fokus stehen eher Fragen nach der ästhetischen Reflexion des Digitalen und nach den Potentialen digitaler Ästhetiken und Praktiken im Kontext postdigitaler Artikulationskulturen (Jörissen 2019).

Gleichwohl sind Ausdifferenzierungen und Weiterentwicklungen des Ansatzes der strukturalen Medienbildung zu beobachten. So analysiert Dan Verständig (2017) in seiner Dissertation über Bildung und Öffentlichkeit im Schnittfeld von Bildungstheorie und öffentlichkeitstheoretischer Forschung «verschiedene Verflechtungen von Code und Sozialem unter Bedingungen digitaler Medialität» mit der Intention, «unterschiedliche Reflexivitätsgrade zu diskutieren, die den digitalen Medien einerseits und der darunter liegenden Infrastruktur andererseits eingeschrieben sind» (Verständig 2017, 237). In der Conclusio hebt Verständig u.a. hervor, dass «Algorithmen bei der Herstellung von Sichtbarkeit von zentraler Bedeutung 
sind», insbesondere «entlang unterschiedlicher Kommerzialisierungslinien des Internet» (ebd., 241). Es reiche nicht aus, lediglich den Code offenzulegen; es sei erforderlich, auch infrastrukturelle Betrachtungen hinzuziehen, um verschiedene Abhängigkeiten und Dynamiken von Code erfassen zu können (ebd. 239). Die Arbeit von Verständig unterstreicht die Notwendigkeit, auch strukturelle Veränderungsprozesse zu untersuchen und dies im Kontext einer pädagogischen Medienkritik zu berücksichtigen (Verständig 2017, 81).

$\mathrm{Zu}$ einer kritischen Medien- und Gesellschaftsanalyse gehört auch das Thema (Medien und soziale Ungleichheit). Hierzu gab es in der Vergangenheit in der Medienpädagogik verschiedene Analysen, insbesondere im Kontext von Medienaneignungs- und Mediensozialisationsstudien (u.a. Niesyto, Meister, und Moser 2009). Dabei zeigte sich, dass Formen sozialer Ungleichheit und Benachteiligung vor allem dann sichtbar werden, wenn lebenslagenbezogene Ressourcen nicht ausreichen, um Medien aktiv und reflexiv für die eigene Lebensbewältigung und Persönlichkeitsbildung zu nutzen. In der Forschung wird zwischen einem (first-level digital divide) (Unterschiede im Zugang zu digitalen Medien) und einem (second-level digital divide> (unterschiedliche Nutzungspraktiken) differenziert.

In einer Zusammenschau bisheriger Studien halten Verständig, Klein, und Iske (2016) als «weitgehend unstrittig» fest, «dass sowohl die Möglichkeiten des Zugangs zum Internet (und zum Mobile Web) als auch dessen Nutzung ungleich verteilt sind und sich gerade auch sozial-strukturelle Ungleichheiten im Internet reproduzieren» (ebd., 51). Mit Blick auf neuere technologische Veränderungen im Internet analysiert die Autorengruppe einen (zero-level digital divide〉, der die technologisch-strukturale Ebene des Software Codes betont:

«Fragen des Zugangs zu und der Nutzung von Informationen spielen dabei eine ebenso zentrale Rolle wie Fragen nach einer möglichen technologisch-infrastrukturellen Vorstrukturierung von Inhalten, Kommunikations-, Kooperations- und Partizipationsmöglichkeiten. 〈Code〉 kann in dieser Perspektive gerade nicht als 〈neutral〉 verstanden werden.» (ebd., 53) 
Im Ergebnis zeigt sich, «dass digitale Spaltungen und Ungleichheiten sowohl entlang existierender sozialer (offline) Ungleichheiten als auch entlang neuer Medientechnologien und deren Nutzungsweisen verlaufen» (ebd.). In medien- und gesellschaftskritischer Perspektive bedeutet dies, auf die genannten strukturellen Begrenzungen hinzuweisen und sich bewusst zu sein, dass ohne die einschneidende Veränderung entsprechender Rahmenbedingungen auch Formen einer milieusensiblen Medienpädagogik ihre Grenze hat.

Hier kommen wir zu einem Punkt, der über Fragen ungleich verteilter Ressourcen hinausgeht: es geht um übergreifende Strukturprinzipien, die die Entwicklung der heutigen Gesellschaft entscheidend beeinflussen. In einem Beitrag über Medienpädagogik und digitaler Kapitalismus habe ich strukturelle Ähnlichkeiten von digitalen und kapitalistischen Prinzipien herausgearbeitet (siehe Niesyto 2017, 16-19):

- Die Quantifizierung von Sachverhalten: Die Messbarkeit von Prozessen, Kostenfaktoren, Tauschwerten, Profitraten korrespondiert - in Verbindung mit einem quantitativen Wachstumsdenken - auf das Beste mit der numerischen Repräsentation von Daten verschiedenster Art.

- In Verknüpfung mit dem digitalen Strukturprinzip der Augenblicklichkeit können in diesen quantifizierenden Prozessen z.B. im Hochfrequenzhandel an den Börsen bei Transaktionen riesige Kapitalgewinne in kürzester Zeit erzielt werden.

- Die Miniaturisierung und Modularisierung digitaler Bausteine sind wichtige technologische Voraussetzungen, um den Arbeits- und Materialaufwand zu reduzieren, die Fertigungstechnik und Logistik bei der Maschine-zu-Maschine-Kommunikation zu informatisieren (Industrie 4.0) und um die Mensch-Computer-Schnittstelle in Richtung Mensch 2.0 / Künstliche Intelligenz zu forcieren.

Des Weiteren passen die digitalen Strukturprinzipien der Variabilität und der Simulation sehr gut zu kapitalistischen Strukturprinzipien, die auf permanente Anpassungsfähigkeit, Effizienz und ökonomische Verwertbarkeit abzielen. Digitale Vernetzung und Interaktivität ermöglicht die ständige und flexible Verfügbarkeit von Menschen und Maschinen. Die flexible Verfügbarkeit ist wesentlich, um als 〈global player〉 Kapitalakkumulation 
in Richtung Kapitalkonzentration und Monopolbildung vorantreiben zu können. Hinzu kommt die neue Entwicklung, auf die Staab (2019) aufmerksam macht: «Klassische Monopolunternehmen agieren auf Märkten; die Leitunternehmen des digitalen Kapitalismus hingegen sind Märkte» (ebd., 30). Staab nennt diese neuartige Marktkontrolle «proprietäre Märkte» (ebd., 32).

Die Vermessung nahezu aller Lebenswelten und des eigenen Körpers offerieren die Optimierung des Alltags und des Selbst, haben allerdings einen harten kapitalistischen Kern: die Erschliessung neuer Absatzmärkte, die Ökonomisierung immer weiterer Lebensbereiche sowie eine umfassende Kontrolle und Überwachung der Menschen (Zuboff 2018). Gerade im Hinblick auf Medienkritik sind technologiegetriebene Zukunftsmodelle vom gesellschaftlichen Zusammenleben, die die Vielschichtigkeit des Menschseins letztlich der Präzision algorithmischer Berechnungen unterordnen, kritisch zu bewerten. Dies ist eine der elementaren Fragen, die auch in Bildungskontexten zu thematisieren ist. Es geht schlichtweg um die Frage, ob weiterhin eine Pluralität von Erkenntnisweisen von Wirklichkeit und gesellschaftlichen Entwicklungspfaden existieren wird - oder ob Verengungen auf binäre Modi des Weltverstehens und damit verknüpfte gesellschaftliche Leitbilder forciert werden (Niesyto 2017, 20).

In diesem Zusammenhang geht es auch um die Frage nach alternativen Entwicklungspfaden. Derzeit mangelt es an Überlegungen, die nicht nur die Problemfelder verschiedener Formen des digitalen Kapitalismus analysieren, sondern auch gesellschaftlich-ökonomische und mediale Alternativen konkret aufzeigen. Hier steht die Medienpädagogik vor der Aufgabe, sich erheblich stärker auf inter- und transdisziplinäre Diskurse mit anderen Disziplinen und zivilgesellschaftlichen Initiativen einzulassen, z.B. um Fragen einer Gemeinwohl-Ökonomie und Perspektiven einer Suffizienzpolitik auf bildungs- und medienbezogene Fragestellungen zu beziehen.

Dies betrifft auch grundsätzliche Fragen der künftigen Gestaltung des Bildungswesens. Aktuell dominieren funktionalistische Digitalisierungskonzepte in der Bildungspolitik, die auf die Optimierung bestehender Strukturen abzielen. Dass z.B. Schule grundlegend auch anders gestaltet werden könnte (inhaltlich, formal, architektonisch, sozialräumlich) als basierend auf aus dem Industrialisierungszeitalter stammenden Modellen, 
blitzt mitunter bei speziellen (Modell-)Projekten auf, ist aber nicht Gegenstand einer tiefgreifenden Reform. Mehr als bedenklich sind in diesem Zusammenhang neuere digitale Medienkompetenzmodelle der EU, die auf funktionale Skills in mehrschichtigen 〈Kompetenzniveaus〉 abzielen - ohne z.B. medienkritische Dimensionen zu berücksichtigen (European Commission 2019).

\section{Subjektverständnis in der Medienpädagogik reflektieren}

In Analysen, die sich mit dem Theorem der medialen Selbstsozialisation auseinandersetzen, wurde bereits deutlich darauf hingewiesen, dass Postulate wie 〈Autonomie〉 und 〈Wahlfreiheit〉 im Medienhandeln vor dem Hintergrund unterschiedlicher sozialer, kultureller und bildungsbezogener Ressourcen kritisch zu hinterfragen sind (Niesyto 2006, 61-62). In der aktuellen Diskussion betonen Valentin Dander und Stephan Münte-Goussar, dass eine starke Subjektorientierung in der Medienpädagogik auf einer Subjektkonzeption beruht, die das «autonome, gestaltende Individuum» in den Mittelpunkt rückt. Dieses Subjektverständnis korrespondiere mit der neoliberalen Ökonomie, die in der Theorie des «Humankapitals» von einem einzigartigen Subjekt ausgehe, «das seine Existenz unternimmt, indem es seine Kompetenzen, Talente und Potenziale als individuelles Vermögen aktiv, frei und vernünftig investiert» (Dander und Münte-Goussar 2018, 251). Die Autoren verweisen auf theoretische Diskurse der letzten Jahrzehnte, die «dem aufklärerischen Subjekt eine Absage erteilen» (ebd., 249). In Zusammenhang mit diesen Diskursen stellt sich auch die Frage, wie Subjekte ihren Eigensinn angesichts kapitalistischer Enteignungsprozesse und Hegemoniebestrebungen behaupten und entwickeln können.

Hierzu ist anzumerken, dass mediale und symbolische Enteignungsprozesse schon seit geraumer Zeit Gegenstand kritischer Analysen waren (und sind), insbesondere im Kontext von Ansätzen der Cultural Studies. Mit Blick auf verschiedene Paradigmen empfahl z.B. Stuart Hall, strukturalistische und kulturalistische Überlegungen zu verknüpfen (Hall 1999). Aktuelle sozialwissenschaftliche Subjektkonzeptionen, die sich nicht auf eine quasi-anthropologische Postulierung individueller Subjektivität und auch nicht «auf eine bloss normative Beanspruchung von Begriffen wie Selbstbestimmung und Mündigkeit» zurückziehen (Scherr 2017, 104), betonen: 
«Das individuelle Empfinden, Denken und Handeln ist in gesellschaftlich vorgegebene soziale Strukturen und Beziehungen eingebettet und ohne deren Berücksichtigung in seiner Entstehung und Entwicklung nicht verständlich.» (ebd., 105)

Dieses sozialwissenschaftliche Subjektverständnis ist anschlussfähig an Ansätze aus der Kritischen Erziehungswissenschaft und Kulturpädagogik (u.a. Krüger 1997, 57-80; Fuchs 2017, 17-21). So war z.B. für den Pädagogen Heinz-Joachim Heydorn Bildung eine «entbundene Selbsttätigkeit», eine «Selbstverfügung», in welcher der Mensch als Subjekt in seine eigene Geschichte eintritt. Heydorn ging von der Dialektik von institutionalisierter Bildung und menschlicher Selbstbildung aus und betonte die Notwendigkeit von Distanzierungsmöglichkeiten und zur Ausbildung von Widerstandspotentialen gegenüber gesellschaftlichen Herrschaftsstrukturen. Bildung im Sinne von «educatio» bedeutet für ihn nicht «Unterweisung», sondern «Hinausführung» als ein Prozess entbundener Selbsttätigkeit (Sünker 2001, 163-67). Dieses Bildungsverständnis impliziert insofern kein überhöhtes Subjektverständnis, als es «Subjektivität begrifflich keineswegs notwendig mit individueller Autonomie im Sinne umfassender Unabhängigkeit von sozialen Bedingungen» gleichsetzt (Scherr 2017, 105). Gleichwohl betont dieses Bildungsverständnis die Möglichkeit zu selbstreflexiven Lern- und Orientierungsprozessen, wie sie auch medienkritische Aktivitäten intendieren.

\section{Medienkritik und normative Fragen}

Die Auseinandersetzung mit dem Subjektverständnis verdeutlicht zugleich die Notwendigkeit der Diskussion normativer Fragen. Zu unterscheiden ist zwischen normativen Setzungen (in Verbindung mit Zieldimensionen bei der Förderung von Medienkritik im Kontext von Medienkompetenz-Konzepten) einerseits und der empirischen 〈Verfasstheit〉 von Subjekten (z.B. den vorhandenen Formen der Weltwahrnehmung/-aneignung, der Weltdeutung, alltäglicher Medienkritik) andererseits. Zu beobachten ist, dass es in diversen medienpädagogischen Konzepten eine Reihe normativer Aussagen bzw. Zieldimensionen in Verbindung mit einer Medienkritik- und 
Medienkompetenzförderung gibt, die oft nicht näher ausgeführt und begründet werden, z.B. 〈humane Gesellschaft), 〈sozial verantwortliches Handeln〉, 〈souveränes Leben〉. Hier stellt sich die Frage, wie Medienpädagogik diese Begriffe inhaltlich konkretisiert und damit verbundene Fragen zum Gegenstand von Bildungs- und Orientierungsprozessen macht. Dies ist eine anspruchsvolle Aufgabenstellung und ist nicht mit einfachen, kanonförmigen normativen Leitlinien zu leisten.

Um es an einem Beispiel etwas zu verdeutlichen: Bezüglich «sozial verantwortlichen Handelns〉 in Verbindung mit ethischen und moralischen Kriterien gibt es z.B. entwicklungsbezogene Konzepte zur Medienkritik (Tulodziecki und Grafe 2018; Ganguin und Sander 2015), die sich auf die Stufenmodelle zur kognitiven Entwicklung (Piaget 1972) und zur Moralentwicklung (Kohlberg 1974) beziehen. Hier zeichnet sich ein Desiderat für weitere Studien ab. So wären z.B. Forschungsbefunde zur Medienkritikfähigkeit von Kindern und Jugendlichen auch im Lichte aktueller entwicklungspsychologischer Theorien und Befunde zu reflektieren. Auch Theorien und Befunde zu emotional-affektiven, motivationalen und sozialen Aspekten von Entwicklung sollten berücksichtigt werden, ebenso wie Netzwerk-Modelle und sozialkonstruktivistische Theorien.

Nicht zuletzt geht es bei normativen Fragen auch um grundlegende Ziele von Bildung und Erziehung. Der in letzter Zeit wieder öfters zitierte Wolfgang Klafki (1985) mag mit den Zielkategorien Selbstbestimmung, Mitbestimmung und Solidarität auf einer allgemeinen Ebene eine Orientierung bieten. Diese Kategorien bleiben aber sehr abstrakt, wenn sie nicht auf aktuelle gesellschaftliche Entwicklungen und Problemlagen bezogen (in Klafkis Worten: auf «epochaltypische Schlüsselprobleme») und mit Reflexionen zur eigenen Positionierung und zu Handlungsmöglichkeiten verknüpft werden. Wer sich z.B. heute in der Erwachsenenbildung aus medienpädagogischer Perspektive mit Digitalisierung in der Arbeitswelt auseinandersetzt, sollte sich fragen, was denn nun Selbstbestimmung, Mitbestimmung und Solidarität in einer Situation bedeuten, wo viele Menschen Ängste vor Arbeitsplatzverlust haben und sich mit Hinweisen auf Schulungskurse zur Aneignung 〈digitaler Kompetenzen〉 nicht zufrieden geben. Hier sind, auch in medien- und gesellschaftskritischer Perspektive, Impulse einer erwachsenenpädagogischen Medienbildung gefragt, die 
nicht bei der Aneignung von digitalem Funktions- und Verfügungswissen stehen bleiben. Denkbar sind z.B. Zukunftswerkstätten in lokalen Kontexten, die gemeinsam mit interessierten Erwachsenen gestaltet werden, um sich über die sozio-technischen Veränderungen in der Arbeitswelt auszutauschen, auch Befürchtungen und Ängste zu artikulieren und gemeinsam nachzudenken, wie man künftig leben und arbeiten möchte und wie man dies kooperativ, auch in Verbindung mit konkreten Projekten, angehen könnte. Ein solches Herangehen ist etwas anderes als Kurse zu (E-Learning〉 oder zu 〈Erwachsenenbildung 4.0〉 anzubieten. Es geht um wert- und handlungsbezogene Fragen, wie Menschen ihr Leben - auch in digital mediatisierten Welten - gestalten möchten.

\section{Medienkritik und pädagogisches Handeln}

Mit Blick auf pädagogisches Handeln haben sich in den letzten Jahren unterschiedliche Konzepte zur Medienkritik auf der Grundlage entwicklungsbezogener, sozial-ästhetischer, technikkritischer, systemtheoretischer, macht- und diskurstheoretischer Ansätze entwickelt (Niesyto und Moser 2018). Kinder und Jugendliche wachsen in konkreten gesellschaftlichen Kontexten auf. Diese Kontexte sind zu analysieren und zu bewerten, auch im Hinblick darauf, ob sie für das Heranwachsen förderlich sind oder nicht. Nach Dieter Spanhel umfasst medienpädagogisches Handeln in Form einer «Kontextsteuerung» des Medienhandelns drei aufeinander abgestimmte Aufgaben: die Begrenzung medialer Sinnorientierungsangebote (Rahmensetzung), Freiräume für selbstbestimmtes Medienhandeln ( $\mathrm{Au}$ tonomiegewährung) und medienpädagogische Begleitung (Medienkompetenzförderung) (Spanhel 2018, 119). Die spannende Frage ist, nach welchen Kriterien die Rahmensetzung erfolgt und wie pädagogisches Handeln im Spannungsfeld von Aushandlungs- und Grenzsetzungsprozessen solche Rahmensetzungen begründet und entwickelt - und zwar so, dass die Perspektive und die Rechte der Kinder und Jugendlichen gewahrt bleiben. ${ }^{7}$

Pädagogische Medienkritik ist nicht nur altersangemessen und milieusensibel zu gestalten (Niesyto 2018,69), sondern im Sinne einer kritischen

7 Siehe hierzu auch die Dokumentation der Fachtagung zum Thema Kinder, Rechte, Medien (2018); https://www.id-tagung.de/ 
Medienpädagogik auch als politisch-kulturelle Medienbildung zu entwickeln. «Kein Mensch» - so der Sozialphilosoph Oskar Negt - «wird als politisches Wesen geboren; deshalb ist politische Bildung eine Existenzvoraussetzung jeder friedensfähigen Gesellschaft» (Negt 2010, 13). Hierzu gehören Wissensbestände, Erfahrungen und die Bereitschaft, das eigene Denken und Handeln immer wieder auf soziale, gemeinschaftliche und gesellschaftliche Kontexte zu beziehen: «Auch in der handlungsorientierten Medienpädagogik geht es nicht nur um differenzierte Wahrnehmung, Deutung, Analyse und Reflexion, sondern auch um veränderungsorientierte Interventionsformen» (Hug 2011, 6).

Medienkritik ist in dieser Perspektive wesentlicher Teil einer politischkulturellen Medienbildung, die ein weites Politikverständnis zur Grundlage hat, welches nicht auf Formen institutionalisierter Politik reduziert ist. Die Kritik am digitalen Kapitalismus und auch die manipulative Verwendung von Daten für politische und andere Zwecke gehört zu den Aufgaben der Medienkritik. Politisch-kulturelle Medienbildung verknüpft dabei alltags- und gesellschaftsrelevante Themen (im Sinne eines weiten Politikverständnisses) mit unterschiedlichen Formen medialer Artikulation und Kommunikation (medienkulturelle Perspektive).

Wichtig ist auch, wie strukturelle Zusammenhänge und Wirkmechanismen der Digitalisierung anschaulich und praxisnah so verdeutlicht werden können, dass Fragen des digitalen Wandels auf der Basis eigener Erfahrungen reflektiert werden können (siehe z.B. das Konzept 〈Digital Citizenship〉, das Heinz Moser unter Bezug auf die amerikanischen Medienpädagoginnen Thoman und Jolls aufgreift; Moser 2019, 222-27). Bisherige Konzepte zur Medienkritik sind oft rationalitätslastig und betonen zu wenig das Zusammenwirken von kognitiven mit emotional-affektiven, sozialkommunikativen und ästhetischen Fähigkeiten. Gerade in handlungsorientierten Projekten besteht die Chance, aus dem Modus der Produktion mit Medien heraus Möglichkeiten für Reflexionsanlässe anhand der Auseinandersetzung mit den selbst erstellten Materialien zu eröffnen (Niesyto 2018, 68-69). Kinder und Jugendliche, die in bildungsbenachteiligenden Milieus aufwachsen, können in handlungsorientierten Medienprojekten anschaulich und praxisnah Medienkompetenzen und Medienkritikfähigkeit erwerben (siehe auch (Kutscher und Farrenberg 2017). Es ist wichtig, dies immer 
wieder zu betonen: medienpädagogische Konzepte sind zielgruppenorientiert zu entwickeln und Theorie-Praxis-Zusammenhänge sind wesentlich, um auch medienpädagogische Theoriebildung und Forschung zu befruchten.

\section{Literatur}

Aßmann, Sandra, Niels Brüggen, Valentin Dander, Harald Gapski, Gerda Sieben, Angela Tillmann, und Isabell Zorn. 2016. «Digitale Datenerhebung und -verwertung als Herausforderung für Medienbildung und Gesellschaft. Ein medienpädagogisches Diskussionspapier zu Big Data und Data Analytics». http:// www.gmk-net.de/fileadmin/pdf/bigdata_diskussionspapier_gmk_kbom.pdf.

Aufenanger, Stefan. 2018. "Media Panics - zur Rhetorik bewahrpädagogischer Positionen in den Medien». In Medien als Alltag. Festschrift für Klaus NeumannBraun, herausgegeben von Ulla Autenrieth, Daniel Klug, Axel Schmidt, und Armulf Deppermann, 462-82. Köln: Herbert von Halem Verlag.

Baacke, Dieter. 1997. Medienpädagogik. Tübingen: Niemeyer.

Barberi, Alessandro, und Christian Swertz. 2017. "Strukturwandel der Öffentlichkeit 3.0 mit allen Updates». In Der neue Strukturwandel von Öffentlichkeit. Reflexionen in pädagogischer Perspektive, herausgegeben von Ulrich Binder und Jürgen Oelkers. Weinheim/Basel: Beltz Juventa Preprint. https://www. pedocs.de/frontdoor.php?source_opus=15610.

Brüggen, Niels. 2017. Medienaneignung und ästhetische Werturteile: Zur Bedeutung des Urteils 〈Gefällt mir!) in Theorie, Forschung und Praxis der Medienpädagogik. München: kopaed.

Damberger, Thomas. 2019. «Antigott. Zur Exklusion des Humanen im Digitalzeitalter». http://damberger.org/wp-content/uploads/2019/02/DambergerAntigott-2019.pdf.

Dander, Valentin. 2018. «Medienpädagogik Im Lichte | Im Schatten Digitaler Daten. Manteltext». MedienPädagogik: Zeitschrift für Theorie Und Praxis Der Medienbildung (Zurück in die Zukunft), 1-134. https://doi.org/10.21240/mpaed/diss. vd.ol.X.

Dander, Valentin, und Stephan Münte-Goussar. 2018. «Die Medienkritik, die wir meinen: Medienpädagogische Medienkritik mit Foucault und Marx». In $\mathrm{Me}$ dienkritik im digitalen Zeitalter, herausgegeben von Horst Niesyto und Heinz Moser, 247-59. München: kopaed.

European Commission. 2019. «The Digital Competence Framework 2.0». https:// ec.europa.eu/jrc/en/digcomp/digital-competence-framework.

Fuchs, Max. 2017. «Brauchen wir eine kritische Kulturpädagogik?» In Kritische Kulturpädagogik, herausgegeben von Max Fuchs und Tom Braun, 11-33. München: kopaed. 
Ganguin, Sonja. 2006. «Das 〈Kritische〉 an der Medienkritik». In Medienkritik heute. Grundlagen, Beispiele, Praxisfelder, herausgegeben von Horst Niesyto, Matthias Rath, und Hubert Sowa, 71-86. München: kopaed.

Ganguin, Sonja, und Uwe Sander. 2015. «Zur Entwicklung von Medienkritik». In Medienpädagogik - ein Überblick, herausgegeben von Friederike Von Gross, Dorothee M. Meister, und Uwe Sander, 229-46. Weinheim und Basel: Beltz Juventa.

Grimm, Petra, und Oliver Zöllner. 2018. Mensch Maschine. Ethische Sichtweisen auf ein Spannungsverhältnis. Stuttgart: Franz Steiner Verlag.

Hall, Stuart. 1999. «Die zwei Paradigmen der Cultural Studies». In Widerspenstige Kulturen. Cultural Studies als Herausforderung, herausgegeben von Rainer Winter und Karl H. Hörning, 13-24. Frankfurt/Main: Suhrkamp.

Hepp, Andreas. 2018. «Von der Mediatisierung zur tiefgreifenden Mediatisierung. Konstruktivistische Grundlagen und Weiterentwicklungen in der Mediatisierungsforschung». In Kommunikation - Medien - Konstruktion. Braucht die Mediatisierungsforschung den Kommunikativen Konstruktivismus?, herausgegeben von Jo Reichertz und Richard Bettmann, 27-45. https://doi.org/10.1007/978-3658-21204-9_2.

Holzwarth, Peter. 2012. «Menschen verändern Bilder - Bilder verändern Menschen. Dossiers Medien im Kontext. Digital Learning Center». Pädagogische Hochschule Zürich. https://phzh.ch/globalassets/phzh.ch/fachbereiche/medienbildung/dossier_bildmanipulation_2012.pdf.

Hug, Theo. 2011. «Sondierungen im Spannungsfeld von Medienaktivismus und handlungsorientierter Medienpädagogik». Medienimpulse 49 (2). https://journals.univie.ac.at/index.php/mp/article/view/mizo8.

Jörissen, Benjamin. 2015. «Transgressive Artikulation: Ästhetik und Medialität aus Perspektive der strukturalen Medienbildung». In Medienkultur und Bildung, herausgegeben von Malte Hagener und Vinzenz Hediger, 49-64. Frankfurt am Main: Campus.

Jörissen, Benjamin. 2019. «Digital/Kulturelle Bildung: Plädoyer für eine Pädagogik der ästhetischen Reflexion digitaler Kultur». Kulturelle Bildung online. https://www.kubi-online.de/artikel/digital-kulturelle-bildung-plaedoyer-paedagogik-aesthetischen-reflexion-digitaler-kultur.

Klafki, Wolfgang. 1985. Neue Studien zur Bildungstheorie und Didaktik. Beiträge zur kritisch-konstruktiven Didaktik. Weinheim/Basel: Beltz.

Knaus, Thomas. 2017. «Pädagogik des Digitalen. Phänomene - Potenziale - Perspektiven». In Software takes command. Herausforderungen der 〈Datafizierung〉 für die Medienpädagogik in Theorie und Praxis, herausgegeben von Sabine Eder, Claudia Mikat, und Angela Tillmann, 49-68. München: kopaed.

Knaus, Thomas. 2018. «Technikkritik und Selbstverantwortung. Plädoyer für ein erweitertes Medienkritikverständnis». In Medienkritik im digitalen Zeitalter, herausgegeben von Horst Niesyto und Heinz Moser, 91-107. München: kopaed.

Kohlberg, Lawrence. 1974. Zur kognitiven Entwicklung des Kindes. Frankfurt/Main: Suhrkamp. 
Krüger, Heinz-H. 1997. Einführung in Theorien und Methoden der Erziehungswissenschaft. Opladen: Leske + Budrich.

Kübler, Hans-Dieter. 2006. «Zurück zum 〈kritischen Rezipienten`? Aufgaben und Grenzen pädagogischer Medienkritik». In Medienkritik heute. Grundlagen, Beispiele, Praxisfelder, herausgegeben von Horst Niesyto, Matthias Rath, und Hubert Sowa, 17-52. München: kopaed.

Kübler, Hans-Dieter. 2018. «Prämissen und Paradigmen von Medienkritik. Versuch einer Kategorisierung». In Medienkritik im digitalen Zeitalter, herausgegeben von Horst Niesyto und Heinz Moser, 15-31. München: kopaed.

Kutscher, Nadia, und Dominik Farrenberg. 2017. «Teilhabe und soziale Kompetenz durch die Nutzung von digitalen Medien: Herausforderungen für die Kinderund Jugendpolitik. Expertise für den 10. Kinder- und Jugendbericht des Landes Nordrhein-Westfalen». https://www.mfkjks.nrw/sites/default/files/asset/ document/10-kjbnrw-expertise-kutscher_farrenberg_u.a.pdf.

Maurer, Björn, und Selina Ingold. 2018. «Digitale Subtexte lesen und schreiben. Handlungsorientierte Medienkritik im MakerSpace an einer Primarschule». In Medienkritik im digitalen Zeitalter, herausgegeben von Horst Niesyto und Heinz Moser, 193-205. München: kopaed.

Maurer, Björn, und Selina Ingold. 2019. «Mit Making zu mehr digitaler Mündigkeit? Ein Design-Based Research-Projekt an einer Primarschule». In medien + erziehung Heft 4: 44-54.

Meister, Dorothee M., und Uwe Sander. 2017. «Kindliche Medien- und Werbekompetenz als Thema der Medienforschung». MedienPädagogik: Zeitschrift für Theorie Und Praxis Der Medienbildung 3 (Jahrbuch Medienpädagogik), 185-200. https://doi.org/10.21240/mpaed/retro/2017.07.11.X.

Missomelius, Petra. 2018. «Kritik als Cultural Hacking». In Medienkritik im digitalen Zeitalter, herausgegeben von Horst Niesyto und Heinz Moser, 167-77. München: kopaed.

Moser, Heinz. 2019. Einführung in die Medienpädagogik. Aufwachsen im digitalen Zeitalter. Wiesbaden: Springer VS.

Negt, Oskar. 2010. Der politische Mensch. Göttingen: Steidl.

Niesyto, Horst. 2004. Medienpädagogik und soziokulturelle Unterschiede: Langfassung einer Studie auf der Basis von Experten-Interviews in Baden-Württemberg und Rheinland-Pfalz. Ludwigsburg: Pädag. Hochsch.

Niesyto, Horst. 2006. «Medienkritik und Mediensozialisation». In Medienkritik heute. Grundlagen, Beispiele, Praxisfelder, herausgegeben von Horst Niesyto, Matthias Rath, und Hubert Sowa, 53-70. München: kopaed.

Niesyto, Horst. 2017. «Medienpädagogik und digitaler Kapitalismus. Für die Stärkung einer gesellschafts- und medienkritischen Perspektive». MedienPädagogik: Zeitschrift für Theorie Und Praxis Der Medienbildung 27 (Spannungsfelder \& blinde Flecken), 1-29. https://doi.org/10.21240/mpaed/27/2017.01.13.X. 
Niesyto, Horst. 2018. «Medienkritik - Entwicklungsphasen und aktuelle Herausforderungen». In Medienkritik im digitalen Zeitalter, herausgegeben von Horst Niesyto und Heinz Moser, 59-75. München: kopaed. http://www.horst-niesyto.de/wp-content/uploads/2019/08/Niesyto_Medienkritik_2018.pdf.

Niesyto, Horst. 2019. «Ergebnisse des Entwicklungsprojekts dileg-SL - Kernpunkte in teilprojektübergreifender Perspektive». In Digitale Medien in der Grundschullehrerbildung. Erfahrungen aus dem Projekt dileg-SL, herausgegeben von Thorsten Junge und Horst Niesyto, 207-32. München: kopaed. https:// www.ph-ludwigsburg.de/fileadmin/subsites/lb-mpxx-t-ol/Junge/dileg-SL2019-Niesyto_-_Ergebnisse_des_Entwicklungsprojekts.pdf.

Niesyto, Horst, Dorothee M. Meister, und Heinz Moser, Hrsg. 2009. Medien und soziokulturelle Unterschiede. Zürich: Zeitschrift MedienPädagogik 17. https:// doi.org/10.21240/mpaed/17.X.

Niesyto, Horst, und Heinz Moser, Hrsg. 2018. Medienkritik im digitalen Zeitalter. München: kopaed. http://www.horst-niesyto.de/band_medienkritik.pdf.

Niesyto, Horst, Matthias Rath, und Hubert Sowa, Hrsg. 2006. Medienkritik heute. Grundlagen, Beispiele und Praxisfelder. München: kopaed.

Piaget, Jean. 1972. Theorien und Methoden der modernen Erziehung. Wien, München, Zürich: Molden.

Rath, Matthias. 2017. «Medienethik». In Grundbegriffe Medienpädagogik, herausgegeben von Bernd Schorb, Anja Hartung-Griemberg, und Christine Dallmann, 240-47. München: kopaed.

Röll, Franz Josef. 2018. «Öffentlichkeit in postdemokratischen Gesellschaften». In Medienkritik im digitalen Zeitalter, herausgegeben von Horst Niesyto und Heinz Moser, 33-44. München: kopaed.

Scherr, Albert. 2017. «Subjektbildung: Grundlagen, Herausforderungen und Perspektiven». In Kritische Kulturpädagogik, herausgegeben von Max Fuchs und Tom Braun, 99-108. München: kopaed.

Scheuble, Walter, Sara Signer, und Heinz Moser. 2014. «Mediale Grundbildung. Das Modell der Pädagogischen Hochschule Zürich». In Grundbildung Medien in pädagogischen Studiengängen, herausgegeben von Peter Imort und Horst Niesyto, 109-23. München: kopaed.

Schorb, Bernd, und Helga Theunert. 2000. «Kontextuelles Verstehen der Medienaneignung». In Qualitative Kinder- und Jugendmedienforschung. Theorie und Methoden: ein Arbeitsbuch, herausgegeben von Bernd Schorb und Ingrid PausHasebrink, 33-57. München: kopaed.

Seyferth-Zapf, Christian, Maria Seyferth-Zapf, und Silke Grafe. 2019. «Handlungsorientierte Förderung von Medienkompetenz im Umgang mit Propaganda der Gegenwart - deutsch-amerikanische Dialoge in einer digitalen Lernumgebung». In Bewegtbildung denken - Beiträge zu Webvideo und politischer Bildung im Social Web, herausgegeben von Netzwerk bewegtbildung.net. 33-44. Berlin/Bonn. https://www.bewegtbildung.net/handlungsorientiertefoerderung-von-medienkompetenz-im-umgang-mit-propaganda-der-gegenwart-deutsch-amerikanische-dialoge-in-einer-digitalen-lernumgebung/. 
Spanhel, Dieter. 2018. «Medienkritik aus pädagogischer Perspektive. Kritik der medialen Bedingungen des Aufwachsens unserer Kinder und Jugendlichen». In Medienkritik im digitalen Zeitalter, herausgegeben von Horst Niesyto und Heinz Moser, 109-23. München: kopaed.

Staab, Philipp. 2019. Digitaler Kapitalismus. Markt und Herrschaft in der Ökonomie der Unknappheit. Berlin: Suhrkamp.

Sünker, Heinz. 2001. «Bildung». In Handbuch Sozialarbeit Sozialpädagogik, herausgegeben von Hans-Uwe Otto und Hans Thiersch, 2. Aufl., 162-68. Neuwied: Luchterhand.

Treumann, Klaus Peter, Dorothee M. Meister, Uwe Sander, Eckehard Burkatzki, Jörg Hagedorn, Manuela Kämmerer, Mareike Strothmann, und Claudia Wegener. 2007. Medienhandeln Jugendlicher. Mediennutzung und Medienkompetenz. Bielefelder Medienkompetenzmodell. Wiesbaden: Springer VS.

Tulodziecki, Gerhard, und Silke Grafe. 2018. «Medienkritik angesichts von Digitalisierung und Mediatisierung aus handlungs- und entwicklungsorientierter Perspektive». In Medienkritik im digitalen Zeitalter, herausgegeben von Horst Niesyto und Heinz Moser, 125-37. München: kopaed.

Tulodziecki, Gerhard, Silke Grafe, und Bardo Herzig. 2013. Gestaltungsorientierte Bildungsforschung. Bad Heilbrunn: Klinkhardt.

Verständig, Dan. 2017. «Bildung und Öffentlichkeit - eine strukturtheoretische Perspektive auf Bildung im Horizont digitaler Medialität». Dissertation, Ottovon-Guericke-Universität Magdeburg. https://doi.org/10.25673/4919.

Verständig, Dan, Aleksandra Klein, und Stefan Iske. 2016. «Zero-Level Digital Divide: Neues Netz und neue Ungleichheiten». Si:So Analysen - Berichte - Kontroversen. Universität Siegen: 50-55.

Zuboff, Joshua. 2018. Das Zeitalter des Überwachungskapitalismus. Frankfurt/New York: Campus. 